\title{
Der Reis.
}

Von Immanuel Löw.

\section{Sprachliches.}

Der Reis heisst mischnisch, nach beliebter Nominal-

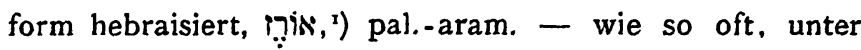
Einfluss des Hebräischen - ebenso (jTer II 4 $4 \mathrm{I}^{\mathrm{d}}, 26$ בשלון

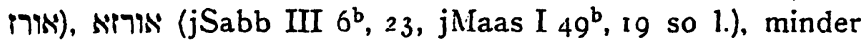

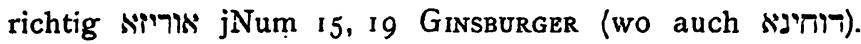
Samaritanisch ist das Wort nicht belegt, denn die LAA

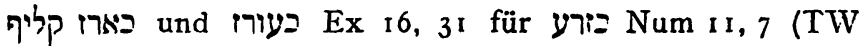
גבורע (ב) sind "nur echt samaritanische Verschreibung \&, die

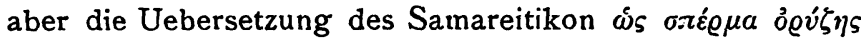
veranlasst hat. ${ }^{2}$ ) Jüdisch-babylonisch ist sims:, $\left.{ }^{3}\right)$ dem das arab. 坊' entlehnt sein dürfte.4) ist gegen Aruch, der diese richtige LA statt פרתיניאי Taan $24^{\mathrm{b}}$ erhalten hat,

1) TBer IV 9, 6. 22, j VI 10b, 10, b $37^{\text {a }}$, Pea 8, 3, Dem 2, I, T II, 47,9 , Švi 2, 7. 10, Chal I, 4; 3, 7. 10;4, 3, 'T II 98, 22, TNed IV 279, 17,

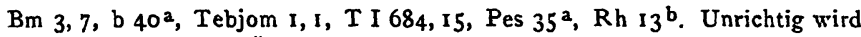
אור (ob für Iris?) TŠvi V 68, 6, j VII $37^{\mathrm{b}}, 28$ sein.

2) S. KonN in MS 38,5 . LXX hat für 7 nicht ǒ $\varrho \zeta \alpha$ trotz KоBur I $277^{a}$ und KRAUSS s. v:

3) Pes $50^{b}$ 1.Z., MsM irrig 171 , $114^{b}$, Gaon Lyck Nr. 45 p. $18^{a}$, Schibbole ha-Leket p. 126 BuBER, irrig Tor. schel Rischonim II 5, 5 ארוזין.

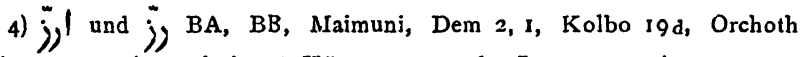
Chajjim $37^{c}$, 5. Armenisch oriz HüBschaANN 369, LAGARDE, gA 24, 224. D. H. Müller, Mehri III $93 \mathrm{Nr}$. 34. $126 \mathrm{Nr}$. I iróz, irehoz. 
nicht Reis, sondern Arsinoë, der grosse Stapelplatz für Getreide. Es heisst "Weizen aus Arsinoë«.') Wellhadsen führt mand. וצריא aus Thesaur. 213,16 an. [Dazu bemerkt NöLdeke brieflich: „Die Stelle SR I 213 , ברמו עבארא מסדרא 15-16 lautet: Sei ein wahrer Nasoräer

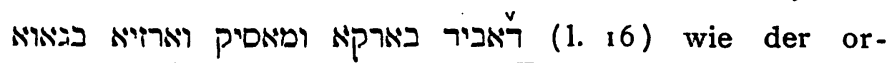
dentliche Ackersmann, der die Erde bearbeitet und in ihr Saaten emporbringt (aufwachsen lässt). ו'אר ist auf keinen Fall "Reis\&; hier wäre eine spezielle Frucht schlecht angebracht und auch die Form warzé passte weder zu ö $\varrho v \zeta \alpha$ noch zu vrīhi. וארזיא ist ohne Zweifel das pers. $j \bar{j}$, das merkwürdigerweise auch im $\mathrm{Np}$ das anlautende, behalten hat, aber daneben das re-

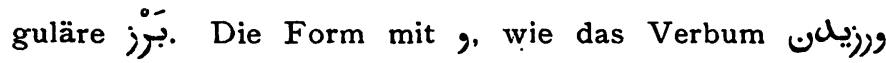

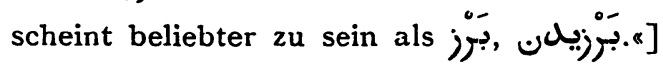

Als syrische berechtigte Form kann ich nur ho; anerkennen. ${ }^{2}$ ) Das aus Bar $\mathrm{K} \in \mathrm{pha}$ angeführte einmalige $h=;\left.\right|^{3)}$ ist die jüdisch-babylonische Form des Wortes, während heisl PSM 93 (und natürlich auch im kleinen psm 533) nur das transkribierte griechische Wort ist.

Auch $\eta_{1}$; BHebr, ZDMG 40, 45 $1^{4}$ ) bei Brock. ist zu

r) Vgl. שב חיטי יארופייתא Gitt 70a, 2. Arsinoë Stapelplatz für Getreide: Wessely, Die Stadt Arsinoc̈ ${ }_{4} 8$.

2) Peš., Galen-Uebers., BHebr (List of plants 97). Honein (DBB 668), $\mathrm{KDg}$, Sindban, PSM 3846, DBB 824. 1881. CARD. 465 : П̆ ;. [NÖLDEKE brieflich: „Für he; habe ich noch Thomas Marg. 269, 22. 270, I, wonach man in Dèlum und Gēlūn das Brot aus ḩ⿻; backte, da es dort keinen Weizen und keine Gerste gebe. Ich denke, noch jetzt wird in den Ländern südlich vom kaspischen Meer Reis gebaut. «]

3) Ueber hici| DBB 285 s. Krauss II 26.

4) [NöLdzKE brieflich: „Für das von MORAles hier gegebene $\eta$; hat Budge's Ausgabe The laughable stories S. 123 Nr. 596 zweimal $ן$. In der arabischen Vorlage, die BHebr benutzt haben wird, stand gewiss j).“] 
bezweifeln, ol,wohl es nach ns hi: rizá, Mackeas, möglich ist. $h^{\circ} \mathrm{K}$ hei PSM 3846.3876 ist falsch.

Alle diese Formen gehen aufs Griechische zurück.

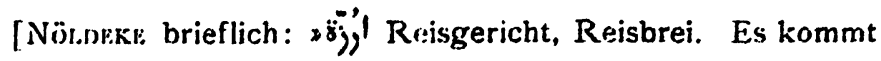
Tab. 1, 2127,17 bei einem Ereignis des Jahres 19 d. $\mathrm{H}$. vor. So in einem Gedicht des 3. Jhrh. d. H. Mas'ūdi 8. 401. Aus früh-abbasidischer Zeit hat Baihaqi (ed. Scrwslly) 2 Stellen mit $\ddot{8 j}_{\text {j) }}$ Reisgericht 554, 13, wo der Koch dem Barmekiden Jahjã dies Gericht mit Nafta statt mit KokosOel bereitet, und $59 \mathrm{I}$ ult. 592, 2.

j) als Nebenform von $\ddot{j}^{\prime}$ hat schon Gauhari. Er hat auch die merkwürdige Form $\stackrel{\text {; }}{;}$, und diese wird belegt von Gawālíqī, ZDMG 33,217. Diese Form mit $n$ macht es doch fraglich, dass die aramäischen und arabischen Formen aus dem Griechischen stammen. Gawāliqii a. O. hat die Formen noch gibt, ist wohl falsch. j) hat $G$. nicht, Lisān hat die beiden Formen auch nicht.)

Das $\zeta$ der griechischen und semitischen Formen deutet auf iranische Vermittlung; da wird ja indisch $h \mathrm{zu}$;

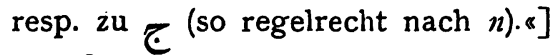

Obwohl schon zur Perserzeit die Reiskultur bis zum Euphrat vorgedrungen war, ${ }^{2}$ ) so kam diese indische ${ }^{2}$ ) Pflanze erst in der Zeit nach Alexander nach Syrien, wie denn der Reis sich - wohl infolge der Schwierigkeit des Anbaus - nur langsam verbreitete und nach Europa erst durch die spanischen Araber kam.3) Nach Gustav Oppert

1) $\mathrm{HEHN}^{6} 488$.

2) Maimuni הזנהגת הבריאות 16 weiss, dass der Reis in Indien gegessen wird.

3) НEHN 6489. 


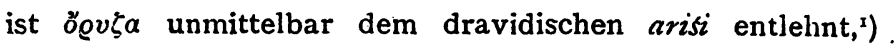
während die ursprüngliche Form vrîhi im pers. بن erscheint, ${ }^{2}$ ) das syrisch transkribiert صنز ergibt.3) [NöLDEkE brieflich: "بَنْ bedarf keines Beleges. Schahname4) (MACAN)

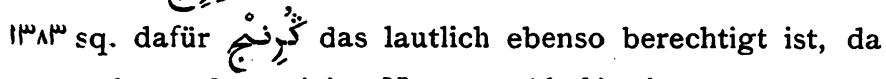
aus anlautendem $v i$ im Np. sowohl $b i$ wie $g u$ entstehen

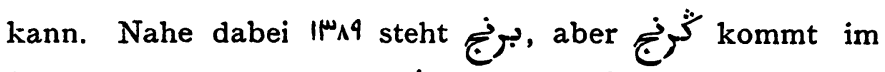
Schahname öfter vor als برنج, und die Gleichberechtigung beider Formen für Firdausi's Land und Zeit wird dadurch gesichert, dass bei Muwaffaq S. 6 paen. s. v. ¡j der Reis

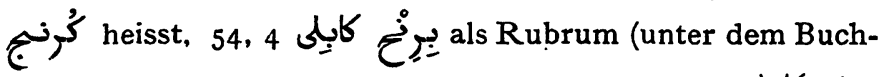
staben b) und darauf noch einmal in derselben Zeile برنج steht. Dieser Form muss meines Erachtens eine indische mit Nasal zu Grunde liegen. Für vrinhi wäre biring, guring durchaus regelrecht; nicht für vrihi.»]

Bisher unerklärt war die Glosse 20 DBB 207 I, PSM. 4458. Das ist verschriebenes temn, temen, das in Mittelarabien jetzt für Reis übliche Wort dunkler Herkunft. 5) [NöLDkKE brieflich: »Das moderne تََّّى ist wohl aus Indien. Auf keinen Fall semitisch. Es ist gebräuch-

1) KOHUT, Studies 398.

2) LAGARDE, gA 24, 224. פלברנג Barzillaj, Jezira 230. 348. פירינג. Tobia Kohen s. unten.

3) $\mathrm{DBB}$ 432. 285 n. 16. Weio $668 \mathrm{PSM}$ 1068.

4) Stellen für letzterer Stelle,.Z. 5 v.u. aber برن. Unsere Schahname-Texte sind nicht so, dass auf solche Dinge irgend Verlass wäre, aber der Text Muwaffaq's ist sehr gut.

5) Vollers, ZDMG 50, 629. Reis in Arabien ZDMG 49, 714. 
lich in 'Irăq und in Arabien selbst. Das Glossar zu Socrn's Diwan ans Centralarabien hat (III 251) timmän Reis, auch

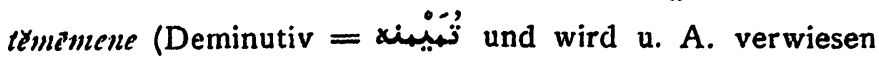
auf Dovgrry 1, $153 \mathrm{temmn}$, river rice from Mesopotamie. De Goeje stellt im Glossar zu den Geographen IV 198 den in Chūzistån üblichen Ausdruck für ein ganz kleines Gewicht تهونة zu diesem Worte. Das war mir nicht wahrscheinlich; aber als ich die zitierte Stelle Muqaddasi 417, 13 nachschlug und da fand, dass Muq. selbst dazu setzt: وأsى الازئ (in Cod. C, der eine andere vom Verfasser selbst gemachte Rezension des Buches gibt: إز اله يسهونها تهونه), musste ich ihm beitreten. De GOEJE zitiert noch Ibn Hauqal 179, 9, aber dies Zitat ist leider falsch. 6 ]

Von fremdsprachlichen Glossen in der rabbinischen Literatur ist zu erwähnen: riso RISip. Pea 8, 3, Svi 2, 7 . ריש Kolbo 19d, Lebusch und Mag. Abr. Or. Ch. 208, 7.

\section{i. Geschichtliches.}

R. Jochanan b. Nūrī, der sich auch sonst für botanischhalachische Spezialitäten interessierte, wollte den Reis unter die Getreidearten eingereiht wissen, konnte aber mit seiner Meinung gegen die Mehrheit der Schriftgelehrten nicht durchdringen. Doch zeigt seine Opposition, dass keine alte, unanfechtbare Praxis für die Klassifikation des Reises vorlag. Er und sein grosser Zeitgenosse $R$. A kiba ${ }^{2}$ ) sind die ersten, deren Name in Verbindung mit dem Reis vorkommt; es ist darum nicht wahrscheinlich, dass man den Reis in Palästina wesentlich früher, etwa schon um die Mitte des ersten vorchristlichen Jahrhunderts angebaut habe. ${ }^{2}$ ) Zum Eingange des ersten Jahrhunderts, wohin uns eine Generation vor $R$. Akiba führt, stimmt. nun sehr gut, wenn Strabo berichtet, man baue auch in Niedersyrien

1) $\mathrm{TBm}$ III 376,25 .

2) J. Enc. s. v. Botany p. 334 . 


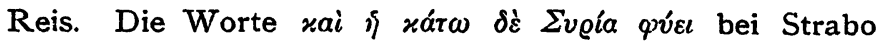
$\mathrm{XV}$ I, 8 hält Hens, wie es scheint mit Recht, für einen Zusatz Strabo's zu dem Berichte des Aristobulos, eines Begleiters Alexander's d. Gr., über den Reisbau in Baktriana, Babylonien und Susis.

Ich kann mich darum der sehr ingeniösen und bestechenden Konjektur WELLHAUSEN's, ${ }^{x}$ ) so gelegen mir ein Beleg für Reis bei Sirach wäre, nicht anschliessen. $\mathrm{Zu}$ dem geschichtlichen Grunde kommt hinzu, dass Reis nicht

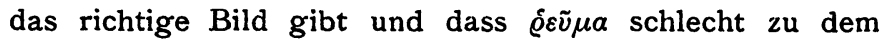
stehenden sumpfigen Wasser passt, in welchem die Reissaat gedeiht.

\section{Sachliches.}

Der palästinische Reis hatte schon in mischnischer Zeit eine besondere Qualität; er war, wie es scheint, von besonderer Weisse, die sonst nur in der Gegend von Antiochien $^{2}$ ) vorkam, doch soll auch der antiochenische Reis rötlicher gewesen sein. ${ }^{3}$ )

Ueber den Anbau des Reises berichtet die Mischnah gelegentlich, man dürfe die Saat im Sabbatjahre wohl einschlemmen - das dürfte ממרס meinen - aber nicht die

1) Wellhausen will nach brieflicher Mitteilung S. Fraenkel's Sir. 39, 13 für Rose: Reis lesen. Ich weiss nicht, ob W.'s Konjektur schon veröffentlicht ist und habe die vorliegenden Bemerkungen über den Reis auf die Mitteilung Fraenkel's hin geschrieben.

[NöLDERE brieflich: „Die Annahme W.'s ist wirklich bedenklich. Der syrische Text stellt manches um. Seine |७や hängen wohl mit der Verwechslung von לבנן Vers 14 zusammen.a]

2) (jHor III $48^{\mathrm{a}}, 45$, Jalk. Prov. 956 aus Vaj. r. 5, 4

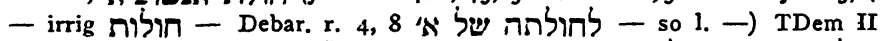
47, 9, jII 22d, ix, irrig RŠ zu Dem 2, s. Weder א חולת noch das daselbst genannte (בורו) בורן ist, trotz Jastrolv 194, identifiziert. Maim. zu Dem 2, 1. Reisbau bei Tiberias: BuHLE, Calendarium Palaest. occonomicum, Göttingen 1785 p. 29.

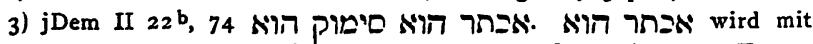
der Marginalnote als Korruptel von אירוביאי LY I 280 und Azulaj, der iרוק erklärt, also an أخضر denkt. 
Blätter abschneiden - מבסחין Švi 2, 10 -, um die Körnerbildung zu befördern. Die Reissaat, sowie die der Gefährten des Reises in der tannaitischen Terminologie: Durra, Hirse und Sesam, reift nicht gleichzeitig, sondern strichweise, darum wird ihr Verhältnis zum Sabbathjahre nicht wie das der Getreidearten nach dem Anfangsstadium der Reife, sondern nach dem Wurzelschlagen bestimmt. Nach anderer Ansicht griff man auf das Wurzelschlagen zurück, weil der durchschnittliche Reifegrad sich beim Reis nicht gut bestimmen lässt. ${ }^{\text {) }}$

Der Schwund für enthülsten Reis wird wie für Weizen auf $2,5 \%$ pro Jahr berechnet, während er sich für Gerste und Durra auf $5 \%$, für unenthülsten Reis, für Emmer und Leinsaat auf $10 \%$ stellt. $^{2}$ )

Der Reis wird enthülst ${ }^{3}$ ) und kommt so in den Handel. Er wird hauptsächlich in gekochtem Zustande gegessen, ${ }^{4}$ ) auch am Pessach, da er für keine Getreideart gilt5) und auch keine eigentliche Brotfrucht ist. Man kennt in $\mathrm{Pa}$ lästina viererlei Arten der Zubereitung ${ }^{6}$ ) durch Kochen, wobei der Aufmerksamkeit nicht entging, dass der gekochte Reis die Wärme auffallend lange hält.7)

Eine eigentliche Brotfrucht ist der Reis, wie gesagt, nicht; doch wurde aus Reismehl, eventuell unter Zusatz

1) Švi 2, 7, RŠ zSt. jII $34^{\mathrm{a}}$, 3. I I, Sifra Behar $105^{\mathrm{c}}, \mathrm{RAbD} z \mathrm{St}$. Rh I $_{3}{ }^{\mathrm{b}}$. nicht „Hülsenfrüchte, die enthülst werden (LEVY), bei RISip. durch ערוגות Beete wiedergegeben. R. Chananel schweben dabei die Beete vor, in welche die Reisfelder durch die Dämme geteilt werden.

2) $\mathrm{Bm} 3,7, \mathrm{~T}$ III $376,23, \mathrm{~b} 40^{2}$.

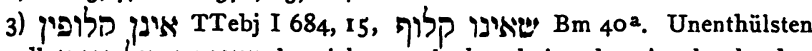
Reis soll שעורה של שור שורז bezeichnen, doch scheint der Ausdruck eher auf die Rispe zu gehen. Pea 8, 3, jTer I 40d, 5 .

4) מבושל Pea 8, 3, jTer I 4rd, 27. משלון אורח jTer das., Tebj ז, 1. ארוזא כר פבשל Gaon Lyck Nr. 45, p $18^{\mathrm{a}}$.

5) Pes $114^{b}$ לת פשורi מבושל : RChan.

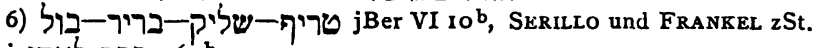

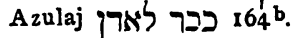

7) פינבא ראורוא בפר jMaas I 49b, I9(so), jSabb III 6b, 23. 
von Weizenmehl auch Brot gebacken, ${ }^{x}$ ) das aber als Mazza nicht verwendet werden durfte. ${ }^{2}$ ) Das aus Reismehl hergestellte Gebäck kann dann wieder gekocht werden. ${ }^{3}$ )

\section{Halachisches.}

Die Halacha zählt den Reis trotz Widerspruches des R. Jochanan b. Nürī nicht zu den Getreidearten, ${ }^{4}$ ) sondern zu den sogenannten Sämereien.5) Er steht den Hülsenfrüchten nahe. $\left.{ }^{6}\right)$ Man zählt ihn nicht zum Getreide, denn sein Teig säuert nicht,7) sondern er geht in Fäulnis über. ${ }^{8}$ ) Er bildet mit Durra, Hirse und Sesam eine stehende Reihe, die als besondere Gruppe halachisch gleicher Behandlung unterliegt $\left.{ }^{9}\right)$ und nicht teighebepflichtig ist. ${ }^{\text {.0) }}$ ) Doch unterwarf man den Reis in Chuzistan, wohl weil er dort die eigentliche Brotfrucht vertrat, der Teighebe. ${ }^{\text {II) }}$

Dem Reis steht Durra am.nächsten, auch syrisch werden L.o9o ho; beisammen genannt. ${ }^{22}$ ) Doch steht Durra, wie die Eulogie über Reis zeigt, um eine Schattierung

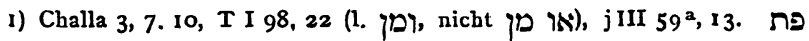

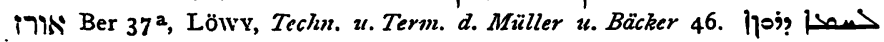
DBB 824, ז עיפת Mech Horrs. 19.

2) Chall 3,7 , Nech Horfm. 19, Pes $35^{a}$.

3) TBer IV 9, 6, jVI 10b, 10.

4) TNed IV 279,17, b $55^{\mathrm{b}}$.

5) TBer IV 9, 6(so), jVI 10b, 10 ivעים

6) Neben קטגיות Mech 8b, 12, Fr. Mech Dt HoffM. I 14 קוטנים jNum 15, 19 und GBGR zSt.

7) ליח so auch jPes II 29b, 14, aber Mech Horrm. 19 durch Pes $35^{\text {a }}$ beeinflusst: חימוץ.

8) לידי סרחנן Mech 8b, MechDt a. O. Sifre I $110,3^{12}, I_{146}$ ) $54^{b}$, jPes II 29b, 14.

9) Švi 2, 7, jII $34^{a}, 10$, Chall 1, 4, Mecb $8^{b}, 12.9^{a}, 12$. Sifre a. O. Mech Dt HorfM. I 14, Pflanzenn. 103.

10) Challa 4, 3, T II 98, 29.

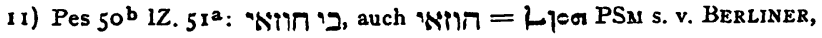
Beitr. \&. Geogr. Bal. 35, JEnc. s. v. Shushan.

12) PS» 3846, A uDo 492. 493. Buchweizen (tatarka) für eine Art Reis gehalten. Pachad Jicchak $\mathfrak{H}^{*}, 184^{\mathrm{a}}$. 
tiefer in der Schätzung, was dem grösseren Nährwerte des Reises oder dem Eindrucke der Opposition R. Jochanan b. Nürr's zuzuschreiben ist. Auch sonst kommt dem Reis eine gesonderte Stellung zu. $\left.{ }^{1}\right)$

Die Halacha hatte Anlass, sich mit dem Reis zu beschäftigen, um die Zugehörigkeit der Saat zu den verschiedenen Deputaten und das Verhältnis der Frucht zum Pessach, ${ }^{2}$ ) zur Teighebe und zu den Eulogien ${ }^{3}$ ) festzustellen, die ihm in den verschiedenen Formen zukommen, in denen er genossen werden kann.

\section{Rabbinisches.}

Raschi gibt in seinem Talmudkommentar אורז durch mil, Hirse wieder, wogegen die Tossafot mit Recht Einspruch erheben. Doch hat Raschi selbst in einem Gutachten die richtige Identifikation: $D$ Die Reisearten, is, die ריש, riz heissen, sind am Pessach nicht verboten, gehören nicht $z u$ den fünf Getreidearten; es ist auch, nach Aussage der Leute aus Reis produzierenden Ländern nicht wahr, dass der Reis, bevor er in den Handel kommt, gekocht werde. ${ }^{4}$ )

Die irrige Nachricht, der Reis werde in seiner Heimat gekocht, bevor er in Handel kommt, wird darauf zurückgehen, dass er nach dem Enthülsen getrocknet oder gedörrt wird, weil er sich sonst nicht zur Versendung eignen würde.

Aus späterer Zeit gibt der Arzt Tobia ha-Kohen die

I) $\operatorname{TAz}$ IV 467,8

2) Pachad Jicchak s. v. 17א. Palaggi Moëd lekol Chaj 2, 7.

3) TBer IV 9, 6, jVI 1ob, Io, b $37^{\text {a }}$. Jehudaj Gaon (MǗlLER, Mafteach p. 29), Gaon Lyck Nr. 45 p. 18 a. Torathan schel Rischonim II 5. Hal. ged. p. 54 und Hildeshemer zur St. Maim HBer 3, 10, Tur u. Sch. Ar. u. Comm. Or. Chajj. 208, 7. Eschkol I 64, Schibbole ha-Leket p. 126. Orchoth Chajjim

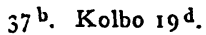

4) Pflanzennamen bei Raschi Nr. 69. 
lateinische und türkische Benennung für Reis: אוריזו oryza und פירינג, in der Sprache der Mischna: ואור.

Aus neuerer Zeit sind einige Zeilen über den Reis bei Baruch Lindau ( 1788 ) zu erwähnen: er schreibt nach, was er in den naturgeschichtlichen Lehrbüchern über den Reis findet, erwähnt den Import aus Italien und Amerika und die Arrak-Bereitung. ${ }^{2}$ )

Nachbemerkung. Reisbau in Aegypten.

Aeltere Nachrichten über den Reisbau in Aegypten hat NORDMEYER zusammengestellt, ${ }^{3}$ ) neuere haben Ascherson und Schweinfurth, die folgende arabische Benennungen haben :

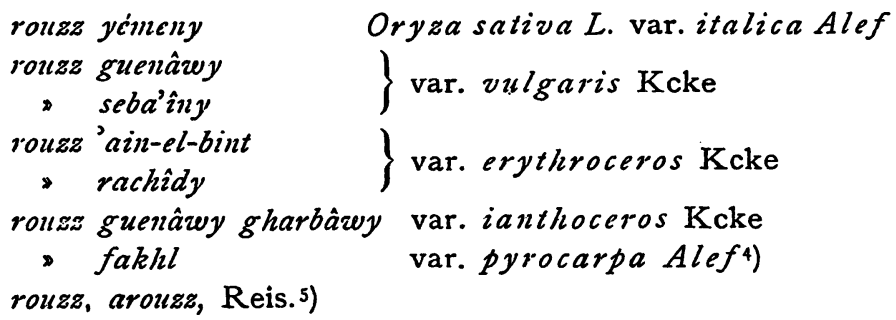

1) Maase Tobija $15^{\mathrm{a}}$.

2) Reschith Limmudim p. $77^{\mathrm{a}}$.

3) Nordmeyer, Calendarium Aegypti oeconomicum 1792. Göttingen. Index s. v. oryza. KÖRNICKE.

4) Ascherson und Schweinfurth, Flore d'Egypte Suppl. 779 nach

5) Das., Flore p. 166. Das. noch djourrai? und choulleykh. Oryza australis ABr. - ruzz Oryza sat. und ruzz el-mâ O. punctata hat R. HartManN, Naturgeschichtlich-medizinische Skizze der Nilländer 178. 